\title{
Anesthetic evaluation of a novel phospholipid-free 1\% propofol microemulsion formulation in dogs
}

Itzcoatl Aquino'

(iD) 0000-0002-6734-8269

Eduardo Gutiérrez-Blanco ${ }^{2}$

(iD) 0000-0002-8370-8367

Luis Ocampo 1

(iD) 0000-0002-0644-8987

Lilia Gutiérrez ${ }^{1}$

(iD) 0000-0002-4823-0388

María J. Bernad-Bernad ${ }^{3}$

Héctor Sumano ${ }^{1, *}$

(iD 0000-0002-8802-5274

${ }^{1}$ Departamento de Fisiología y Farmacología,

Facultad de Medicina Veterinaria,

Universidad Nacional Autónoma de México,

Ciudad de México, México

${ }^{2}$ Departamento de Farmacología

y Anestesiologia Veterinaria,

Facultad de Medicina Veterinaria y Zootecnia,

Universidad Autónoma de Yucatán,

Mérida, Yucatán, México

${ }^{3}$ Departamento de Farmacia,

Facultad de Química,

Universidad Nacional Autónoma de México,

Ciudad de México, México

*Corresponding author:

Email address:

sumano@unam.mx
Accepted: 2019-08-15

Published: 2019-09-30

Additional information and declarations can be found on page 8

(c) Copyright 2019 Héctor Sumano et al.

open access $\mathbf{2}$

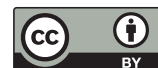

Distributed under Creative Commons CC-BY 4.0

\begin{abstract}
A novel four-week stable experimental formulation of propofol lacking phospholipids (PMICRO) was assessed as an anesthetic agent in dogs and compared to the customary preparation of propofol that contains phospholipids (PMACRO). Forty healthy mixed-breed dogs were included in this study and randomly assigned (blocking by sex and age), to receive $5 \mathrm{mg} / \mathrm{kg}$ of one of these two pharmaceutical propofol preparations, by intravenous injection. Basal and post anesthetic blood biochemical parameters were measured. Also, cardiovascular and respiratory rates were recorded. Onset, duration and recovery from anesthesia, were documented as well. Overall, IV administration of PMICRO induces a short duration anesthesia with rapid recovery in a similar manner to anesthesia achieved with the customary propofol preparation (PMACRO). Because PMICRO lacks phospholipids, it may allow a longer storage time, decreasing the likeliness of accidental administration of contaminated propofol.
\end{abstract}

Keywords: Keywords: Anesthesia, Dog, Microemulsion, Phospholipid-free, Propofol

\section{Cite this as:}

Aquino I, Gutiérrez-Blanco E, Ocampo L, Gutiérrez L, Bernad-Bernad MJ, Sumano $\mathrm{H}$. Anesthetic evaluation of a novel phospholipid-free 1\% propofol microemulsion formulation in dogs. Veterinaria México OA. 2019;6(3). doi: 10.22201/ fmvz.24486760e.2019.3.654 


\section{Introduction}

Propofol is a non-barbiturate, short-acting phenol derivative anesthetic, that is commercially available as a phospholipid macroemulsion for intravenous administration. ${ }^{1-4}$ Undiluted propofol has an 80 to $100 \mathrm{~nm}$ particle size ${ }^{5}$ and hence dilution of the commercial macroemulsion in 5\% glucose is advised, to prevent large particle formation when infusion is prolongued during anesthesia. An infusion pump can be used to deliver the appropriate dose through an IV catheter. 1,6,7 Propofol has a $5.37 \mathrm{~h}$ plasma half-life in dogs, and a large apparent volume of distribution of $6.5 \mathrm{~L} / \mathrm{kg}$. It is rapidly cleared form the body (50 mL/kg/min), It has a high binding affinity to plasma proteins and erythrocytes (97-99\%) and it is metabolized by the liver. $1,6,7$ Due to its high solubility in phospholipids and rapid redistribution, both onset and recovery from anesthesia are smooth and rapidly reached. ${ }^{8}$ It is thus considered one of the safest anesthetic agents available, 9,10 and consequently its use as a pre-anesthetic or an anesthetic has greatly increased in dog surgeries. ${ }^{1,11}$ Continuous IV infusion of undiluted or 5\% glucose diluted propofol allows for the anesthetic procedure to be prolonged as needed, easily changing the depth of anesthesia, and generally attaining uneventful recoveries. ${ }^{1,8}$ However, propofol has disadvantages in its current marketable formulation, which is based on phospholipids (10\% soybean oil, $2.25 \%$ glycerol and 1.2\% purified egg lecithin). ${ }^{12,13}$ Some of these include possibility of fat embolism, hypertriglyceridemia and pancreatitis. ${ }^{12,13}$ Also, it induces transient pain while being injected IV during induction of anesthe$\operatorname{sia}^{4,14,15}$ and a few episodes of anaphylactic reactions have been reported. ${ }^{16-18}$ Moreover, the lipid-based formulation of propofol is physically unstable. ${ }^{13}$ It tends to dissociate and the drug is detached by rupture of the emulsion system. ${ }^{13}$ Consequently, free lipids allow for an increased likelihood of contamination by microorganisms ${ }^{13}$ and the vehicle is capable of supporting rapid bacterial growth. In fact, manufacturers specify that the commercial propofol preparation does not have antimicrobial agents, and that a strict aseptic technique must be maintained when used. Also, prompt administration after opening of the package is advised, as well as subsequently discarding the unused product. ${ }^{13}$ In addition, particulate or bacterial contamination may be difficult to detect macroscopically in an already open emulsion, because propofol injection is opaque. Thus, several studies have been conducted in search for novel propofol phospholipid-free formulations. ${ }^{10,19-21}$

For this study, a propofol microemulsion preparation devoid of phospholipids has been designed. Microemulsions are isotropic mixtures of oil, water and surfactant(s) that are highly soluble and thermodynamically stable. ${ }^{13}$ Anesthetic efficacy, pharmacokinetics and toxicity of propofol microemulsions have been assessed in rodents, and have shown to perform in a similar manner to that of phospholipid based macroemulsions. ${ }^{13,22}$ Nonetheless, similar studies of propofol microemulsions for dogs are still lacking. Thus, the aim of this study was to evaluate the short-term anesthetic features of a phospholipid-free microemulsion of propofol, as compared to the standard propofol macroemulsion. 


\section{Material and methods}

\section{Animals}

This study was approved by the Ethics and Animal Welfare Committee of the Universidad Nacional Autónoma de México, and complied with the official Mexican norm for care and use of laboratory animals NOM-062-ZOO-1999. ${ }^{23}$ Forty mixedbreed feral dogs (20 females and 20 males; 1.5-10 years old), were included in this randomized, prospective, clinical study. Animals were deemed clinically healthy based on an exhaustive physical examination, hematological profiles (urea, creatinine, blood bilirubin, ALT, AST) and medical history. General body condition of dogs was neither too lean nor obese. They were dewormed (Drontal, Bayer Animal Health, Mexico), and an adaptation to housing environment was allowed as for the Guide for the Care and Use of Laboratory Animals, ${ }^{24}$ and food for a period of two weeks was allowed before the start of the trial. Blood samples were obtained a day before and a day after the anesthetic procedure was performed. Food and water were withheld for 10 and 3 hours before anesthesia, respectively.

\section{Pre-anesthetic management and treatments.}

Prior to anesthesia, baseline data for heart rate (HR), respiratory rate (RR), oxygen saturation of hemoglobin $\left(\mathrm{SpO}_{2}\right)$ and rectal body-temperature $(\mathrm{T})$ were obtained. Dogs were subsequently medicated via intramuscular (IM) injection with acepromazine maleate $(0.025 \mathrm{mg} / \mathrm{kg}$ ) (Calmivet, Vetoquinol Mexico, S.A. de C.V., Mexico City, Mexico). As soon as the dogs were sedated, either the right or the left arm was shaved and aseptically prepared, and a 20-gauge IV catheter needle was placed into the cephalic vein, which was then attached to a male PRN connector. Once anesthetized, a proper sized orotracheal cuffed tube was placed in every dog to have rapid access for assisted ventilation if required. Two groups of 20 dogs were randomly formed (blocking by gender and age) as follows: the PMACRO group consisted of dogs which were anesthetized with the commercially available propofol macroemulsion (Diprivan ${ }^{\circledR}$ 1\%, Astra-Zeneca, Mexico City, Mexico) at an approximate IV dose of $5 \mathrm{mg} / \mathrm{kg} .{ }^{11}$ The drug was administered as an initial bolus comprising 60 or $70 \%$ of the total dose in less than $5 \mathrm{~s}$, and a subsequent slow administration of the remaining amount through a period of $60 \mathrm{~s}$. Respiratory rate was continuously monitored to avoid respiratory arrest. In the PMICRO group, the anesthetic procedure was similar to that used for the PMACRO group, but an experimental preparation of $1 \%$ phospholipid-free propofol microemulsion (Propofol Vet ${ }^{\circledR}$, Aranda Pharmaceuticals S.A. de C.V., Querétaro, Mexico) was used. This same phospholipid-free preparation was repeatedly used in dogs within 4 weeks of its manufacturing and showed to remain stable throughout this period.

\section{Manufacturing of the phospholipid-free propofol emulsion}

The PMICRO was prepared by Aranda Pharmaceuticals, sequentially mixing propofol and Crodamol ${ }^{\mathrm{TM}} \mathrm{EO}$ (ethyl oleate), with propylene-glycol, N-metyl-2-pyrrolidone, glycerol-formal and butylhydroxyanisole under Ultra-Turrax stirring $\left(\right.$ IKA $^{\circledR}$-Werke

$\mathrm{GmbH} \&$ Co. KG, Germany), complying with the Mexican norm (NOM-012ZOO-1993), and following good manufacturing practices (GMPs). Particle size of the propofol microemulsions was measured at room temperature (before and after 
dilution), in either sterile $0.89 \%$ saline (SS) or $5 \%$ dextrose (D5W) solutions, by means of a Zetasizer, Zen 3600 (Malvern Panalytical LTD, Malvern, UK) fitted with a $538 \mathrm{~nm}$ laser beam. Final solutions were as follows: basal undiluted PMICRO; $1 \mathrm{~mL}$ PMICRO: $0.01 \mathrm{~mL}$ of SS or D5W; $1 \mathrm{~mL}$ PMICRO: $0.02 \mathrm{~mL}$ of SS or D5W; $1 \mathrm{~mL}$ PMICRO: 0.03 of SS or D5W; $1 \mathrm{~mL}$ PMICRO: $0.04 \mathrm{~mL}$ of SS or D5W; and $1 \mathrm{~mL}$ PMICRO: $0.2 \mathrm{~mL}$ of SS or D5W. The dilution range tested mimicked the conventional dilution of propofol utilized for long-term anesthesia by continuous infusion. 1,3,6,25

\section{Four-week stability test}

A 4-week physical and chemical stability test of the PMICRO was carried out through serial measurements of propofol content by a validated HPLC method 25, 27. All samples were kept in glass vials with rubber-stoppers at $60^{\circ} \mathrm{C}$ with $75 \pm 5 \%$ relative humidity (RH), in a stability chamber (FTL-600, Fine Scientific Instruments, Korea). Sample were analyzed at 0, 1, 2, and 4 weeks.

\section{Trans-anesthetic monitoring}

Values for $\mathrm{HR}, \mathrm{RR}, \mathrm{SpO}_{2}$ and $\mathrm{T}$, were recorded throughout the anesthetic procedure with a multiparameter monitor (LEEX iM8 VET ${ }^{\circledR}$ ). If $\mathrm{SpO}_{2}$ dropped below 90\%, assisted manual ventilation was implemented and registered. Onset (OA), duration (AD), and recovery from anesthesia (RA), considered respectively as the time elapsed between the administration of the anesthetic and the loss of the palpebral and nociceptive withdrawal reflexes, the time that elapsed between the loss and recovery of these reflexes; and the time elapsed between the recovery of reflexes and the moment when the animal attempted or managed to stand up on its own, were also registered.

Serum biochemical and anesthetic parameters between groups were compared by $t$-Student tests, and results were expressed as mean \pm standard deviation (SD). Significant differences were set at $p<0.05$. Physiological parameters were documented as mean and coefficient of variation (CV). Analyses were performed with the IBM SPSSVR Statistics 2.0 software package.

\section{Results and discussion}

The experimental PMICRO preparation was established as a clear and thermodynamically stable microemulsion (an isotropic liquid mixture of a lipid soluble drug -propofol-, water and surfactants). Particle size of the undiluted solution ranged from 80 to $95 \mathrm{~nm}$. However, when mixing $1 \mathrm{ml}$ of PMICRO with any of the tested D5W or SS volumes, the microemulsion was almost immediately transformed (5 min after mixing) into an emulsion, with particle sizes of up to $31,000 \mathrm{~nm}$ (Fig. 1). However, in the storage opaque glass containers, the stability test showed a mean propofol recovery of $98.75 \%$ (range 98.25 to 102\%) after 4 weeks indicating lack of alteration of the pharmaceutical formula. Nevertheless it is important to emphasize that formal stability test for PMICRO is still to be performed. ${ }^{26}$

PMICRO induced clinical anesthesia in all animals. Blood urea, creatinine and bilirubin before and after anesthesia did not differ from basal values in any of the 


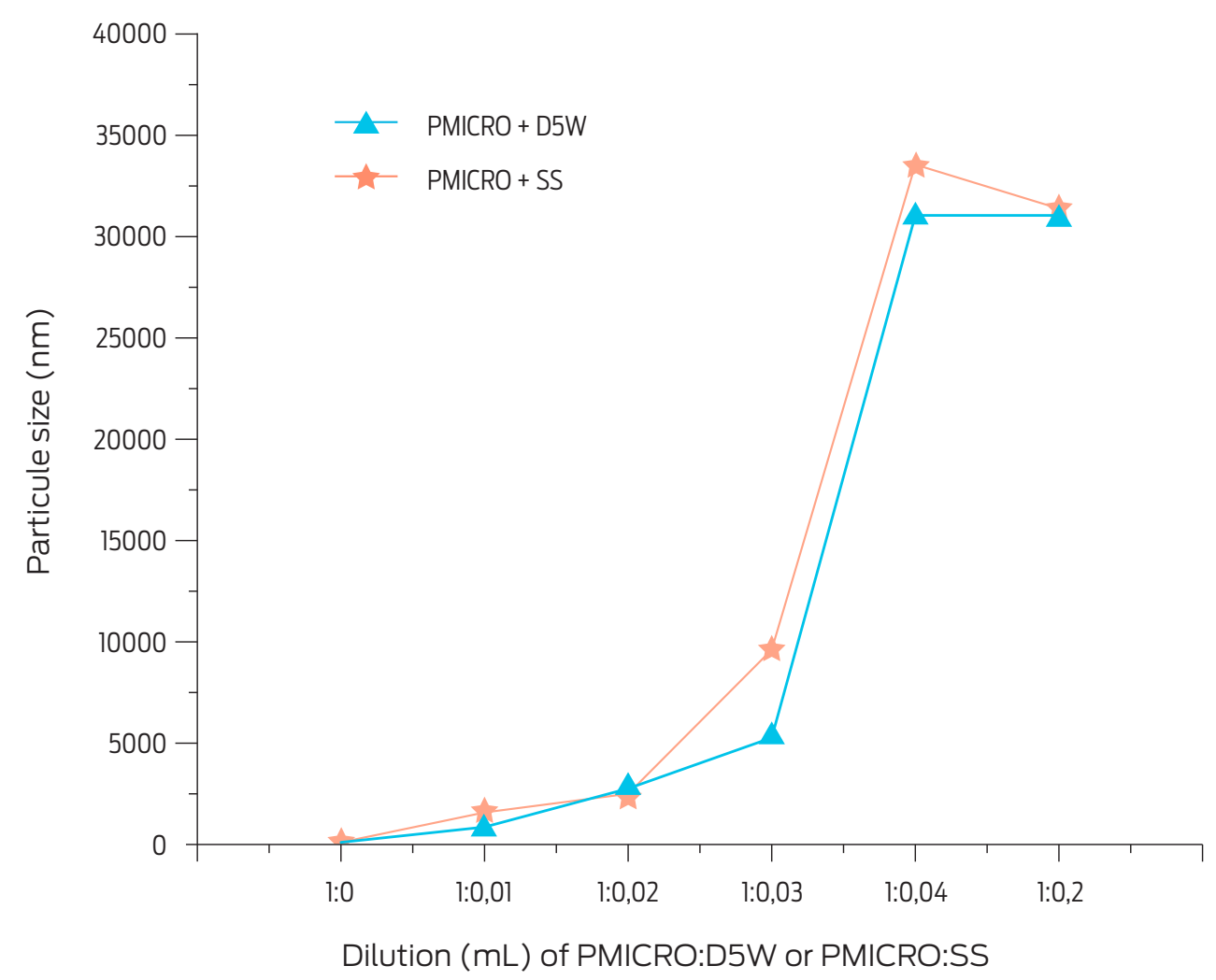

Figure 1. Particle size (nm) of a phospholipid-free 1\% propofol microemulsion (PMICRO) diluted with either sterile 0.89\% saline solution (SS) or 5\% glucose solution (D5W) at different concentrations. Particle size was measured 5 min after dilution.

dogs $(p>0.05)$ and remained within physiologic limits. As shown in Table 1, mean time for OA was $60 \mathrm{~s}$ or less for animals in both treatment groups (PMACRO: $47 \pm 4$ s; PMICRO: $56 \pm 0.3 \mathrm{~s}$ ). However, AD was shorter for the PMACRO group (PMACRO: $12.5 \pm 1.9$ min; PMICRO: $17.5 \pm 2.5, p \leq 0.05$ ). RA was similar between groups (PMACRO: $17.7 \pm 3.4$ min; PMICRO: $19.3 \pm 5.8$ ).

As for physiological parameters, HR values were similar between the PMACRO and the PMICRO groups at time 0 (Table 2). However, five mins after the IV injection, HR decreased to a mean value of $72.4 \mathrm{bpm}(\mathrm{CV}=9.1 \%)$ in the PMACRO group, whereas it increased to $98 \mathrm{bpm}, \mathrm{CV}=8.8 \%$ in the PMICRO group. After this time point, HR values remained steady and within expected ranges throughout the procedure and were similar for all animals in both groups (Table 2).

Respiratory rates were constant and steady throughout the anesthetic procedure and did not differ between groups (Table 3). $\mathrm{SpO}_{2}$ values remained over 92\% through anesthesia in animals in both groups, with few cases exhibiting transient $91 \%$ values in the PMACRO group.

As for $T$, mean recorded values showed minimal variations in both groups ( $38^{\circ} \mathrm{C} ; \mathrm{CV}=1.1 \%$ ) (Table 4). Considering that anesthesia with propofol has been carefully and extensively characterized, including latency and duration action of the anesthetic agent, $\mathrm{HR}, \mathrm{RR}, \mathrm{SpO}_{2}$ and $\mathrm{T}$ are reasonably predictable, and recovery periods are almost constant. 1,27,28 Both the phospholipid-based mixture of propofol and the phospholipid-free preparation used in this study induced clinical anesthesia, where physiological parameters remained within expected values. 
Table 1. Mean duration of anesthetic variables after administration of two propofol treatments

(minutes, mean $\pm \mathrm{SD}$ )

\begin{tabular}{|c|c|c|c|c|c|c|c|c|c|}
\hline Group & \multicolumn{3}{|c|}{ Onset of anesthesia ${ }^{a}$} & \multicolumn{3}{|c|}{ Duration of anesthesia ${ }^{a}$} & \multicolumn{3}{|c|}{ Recovery of anesthesia ${ }^{a}$} \\
\hline PMACRO & $0.7^{b}$ & \pm & 0.2 & $12.5^{c}$ & \pm & 1.9 & $17.7^{b}$ & \pm & 3.4 \\
\hline PMICRO & $0.56^{b}$ & \pm & 0.3 & $17.5^{b}$ & \pm & 2.5 & $19.3^{b}$ & \pm & 5.8 \\
\hline
\end{tabular}

$\mathrm{n}=20$ dogs per group.

PMACRO: phospholipid emulsion of propofol; PMICRO = phospholipid-free microemulsion of propofol 1\%.

a Onset of anesthesia: time elapsed between the administration of the anesthetic and the loss of the palpebral and nociceptive withdrawal reflexes; anesthesia duration: the time that elapses between the loss and recovery of the palpebral and nociceptive withdrawal reflexes); recovery of anesthesia: the time elapsed between the recovery of the palpebral and nociceptive withdrawal reflexes and the moment when the animal attempted or managed to stand up on its own.

$b, c$ Different literals within columns show statistical difference $(p \leq 0.05)$.

Table 2. Mean heart rates registered during anesthesia with two propofol treatments in dogs

\begin{tabular}{|c|r|r|r|r|}
\hline $\begin{array}{c}\text { Time after propofol IV } \\
\text { injection (min) }\end{array}$ & \multicolumn{3}{|c|}{ PMACRO } & \multicolumn{2}{c|}{ PMICRO } \\
\hline & Bpm & CV\% & Bpm & CV\% \\
\hline 0 & 82.8 & 9.1 & 83.4 & 7.5 \\
\hline 5 & 72.4 & 9.1 & 98.5 & 8.8 \\
\hline 10 & 78.7 & 7.4 & 89.4 & 12.8 \\
\hline 15 & 78.4 & 10.6 & 90.2 & 11.6 \\
\hline 20 & 82.7 & 13.6 & 84.9 & 13.3 \\
\hline 40 & 78.1 & 12.7 & 79.8 & 11.9 \\
\hline 40 & 79.5 & 7.7 & 81.7 & 9.9 \\
\hline
\end{tabular}

$\mathrm{n}=20$ dogs per group.

bpm: beats per minute; CV\%: coefficient of variation; PMACRO: phospholipid propofol emulsion; PMICRO: phospholipid-free

$1 \%$ propofol microemulsion.

Table 3. Mean respiratory rates registered through anesthesia with two propofol treatments

\begin{tabular}{|c|r|r|r|r|}
\hline Minute & \multicolumn{2}{|c|}{ PMACRO } & \multicolumn{2}{c|}{ PMICRO } \\
\hline & b/m & CV\% & b/m & CV\% \\
\hline 0 & 22.0 & 9.5 & 22.3 & 9.2 \\
\hline 5 & 21.1 & 11.5 & 23.3 & 8.6 \\
\hline 10 & 19.4 & 9.9 & 23.7 & 9.8 \\
\hline 15 & 20.1 & 11.4 & 23.5 & 10.4 \\
\hline 30 & 19.3 & 10.4 & 22.9 & 9.9 \\
\hline 40 & 21.6 & 11.8 & 23.6 & 12.3 \\
\hline
\end{tabular}

$\mathrm{n}=20$ dogs per group.

$\mathrm{b} / \mathrm{m}$ = breaths per minute; CV\%: coefficient of variation; PMACRO: phospholipid propofol emulsion; PMICRO: phospholipid-free

1\% propofol microemulsion. 
Table 4. Mean body temperature registered through anesthesia with two propofol treatments

\begin{tabular}{|c|c|c|c|c|}
\hline Minute & \multicolumn{2}{|c|}{ PMACRO } & \multicolumn{2}{c|}{ PMICRO } \\
\hline & T( ${ }^{\circ}$ C) & CV\% & T ( ${ }^{\circ}$ C) & CV\% \\
\hline 0 & 38.2 & 1 & 38.3 & 0.8 \\
\hline 5 & 38.2 & 1.1 & 38.2 & 0.5 \\
\hline 10 & 38.2 & 0.5 & 38.3 & 0.5 \\
\hline 15 & 38.2 & 0.5 & 38.2 & 0.5 \\
\hline 20 & 38.1 & 0.9 & 38.1 & 0.8 \\
\hline 30 & 38.1 & 0.5 & 38.1 & 0.4 \\
\hline 40 & 38.1 & 0.5 & 38.2 & 0.4 \\
\hline
\end{tabular}

$\mathrm{n}=20$ dogs per group.

CV\%: coefficient of variation. There were no statistically significant differences when comparing mean values from both groups; PMACRO: phospholipid emulsion propofol; PMICRO: phospholipid-free 1\% propofol microemulsion; $\mathrm{T}\left({ }^{\circ} \mathrm{C}\right)$ : mean temperature in degrees Celsius.

To minimize stress, dogs were pre-anesthesized with acepromazine maleate. Propofol anesthesia onset is dose and injection-speed dependent, but it can also be affected by previous administration of a sedative. Since animals of both treatment groups in this study were medicated with acepromazine prior to anesthesia, it is safe to state that the lack of difference found between groups in OA can be stated independently of the sedative used, and that it can be characterized as smooth and uneventful, as previously described for dogs. ${ }^{1,11}$ Similarly, it has been previously shown that $A D$ after propofol administration is not modified by use of acepromazine.9 Hence, differences found for AD between treatments groups in this study can be attributed to the particular propofol preparation used (Table 1).

Intravenous propofol administration with PMICRO can be used for short-term anesthesia or for quick execution of painful procedures. ${ }^{10}$ If a longer anesthetic time is required with PMICRO, additional propofol could be injected, either directly or aided with an infusion pump capable of delivering approximately 140-180 $\mu \mathrm{g} /$ $\mathrm{kg} / \mathrm{min}$. This will avoid the use of D5W or SS as the zeta potential of the original PMICRO is modified and flocculation and coalescence are favored. It has been observed that the larger the particle size, the lower the anesthetic effect. ${ }^{5}$

Anesthesia with propofol may induce transient apnea and a mild decrease in cardiac output. ${ }^{28}$ In this study, PMACRO transiently decreased HR whereas PMICRO slightly increased HR five minutes after propofol injection, RR was not affected. Subsequent physiologic parameter values were stabilized to match basal recordings. Differences observed at $5 \mathrm{~min}$ after propofol administration may be due to presence of propylene glycol (PPG) in the PMICRO solution, since PPG can moderately alter $\mathrm{HR}$, blood pressure and the efferent sympathetic activity when administered IV in dogs. ${ }^{29}$ Stability of blood $\mathrm{SpO}_{2}$ and $\mathrm{T}$ values throughout the anesthetic procedures for both treatment groups can be related to the short duration of the anesthetic period.

Based on the parameters assessed in this trial, PMICRO can be regarded as a safe anesthetic. Further, the vehicle components used for its preparation did not appear to have any adverse effect. However, as with most drug developments, additional long-term anesthetic and toxicological studies are needed before PMICRO can be used clinically. 
No nanoparticularization of the active principle was attempted considering that manufacturing costs would be considerably increased. Also, release of propofol from nanoparticles may not take place, increasing the potential degree of toxicity of this type of preparation. 11,20,29 In contrast, microemulsions are not considered toxic when particle size surpasses $60 \mathrm{~nm} .{ }^{30}$ In this study, PMICRO was prepared as a microemulsion. However, initial particle size of PMICRO increased when diluted in SS or D5W, since microparticles tended to add up to form larger micelles, giving rise to an emulsion with a modified zeta potential and a flocculation and coalescence that could cause micro-embolisms. ${ }^{5}$

PMICRO was prepared as a phospholipid-free microemulsion to extend shelflife, since phospholipids are prone to support microbial growth. ${ }^{13}$ However, as for any new parenteral preparation, several tests such as presence of pyrogens, chemical reactivity with other drugs and sub-chronic toxicity need to be completed prior to introducing PMICRO for clinical use.

\section{Conclusions and implications}

The phospholipid-free PMICRO emulsion effectively induces short duration anesthesia with rapid recovery in dogs, that is similar to that achieved after administration of the commercially available preparation of propofol emulsified with phospholipids. Since the PMICRO formulation described herein includes chemical vehicles not related to phospholipids, a longer shelf-life is expected. Yet, further studies are warranted to establish the latter.

\section{Author contributions}

H.S. and L.O. conceived the experiment; E. G-B, I.A. and H.S. performed the anesthetic procedures in dogs; M.J. B-B and L.G. evaluated the chemical features of the phospholipid-free propofol, assessed the 4 week-stability studies and analyzed data, respectively. The manuscript was prepared and reviewed by all authors.

\section{References}

1. Sumano H, Pérez N, Izquierdo P, Castellanos J. Anestesia general con propofol en perros mediante infusion continua. Experiencias clinicas. Vet México. 1994;25(3):199-205.

2. Muir W, Gadawski J. Cardiovascular effects of a high dose of romifidine in propofol-anesthetized cats. Am J Vet Res. 2002;63(9):1241-6.

3. Selmi AL, Figueiredo JP, Mendes GM, Lavor LMS, Machado PML. Infusão contínua de propofol em gatos pré-medicados com cetamina-midazolam. Arq Bras Med Veterinária e Zootec [Internet]. 2005;57(3):295-9 [cited 2016 Nov 27]. Available from: http://www.scielo.br/scielo.php?script=sci_arttext\&pid=S0102-09352005000300003\&lng=en\&nrm=iso\&tlng=pt

4. Sim JY, Lee SH, Park DY, Jung JA, Ki KH, Lee DH, et al. Pain on injection with microemulsion propofol. Br J Clin Pharmacol. 2009;67(3):316-25. 
5. Borwankar RP, Lobo LA, Wasan DT. Emulsion stability - kinetics of flocculation and coalescence. Colloids and Surfaces. 1992;69(2-3):135-46.

6. Adams H. Veterinary pharmacology and therapeutics. 9th ed. lowa: Wiley-Blackwell; 2001.

7. Sumano $\mathrm{H}$, Ocampo L. Manual de farmacología clínica para pequeñas especies. 4th ed. Mexico: Imagen Editorial Yire; 2009.

8. Ezquerra J, Vives M. Anestesia práctica de pequeños animales. 1st ed. Madrid: McGraw Hill-Interamericana; 1992. 200 p.

9. Marsico F, Cediel R, Gómez de Segura IA, Tendillo FJ, Criado AB. Un nuevo anestésico intravenoso: propofol: Clínica Vet pequeños Anim [Internet]. 1991;1 1(4):0231-9. Available from: http://ddd.uab.cat/record/69982

10. Tsagogiorgas C, Theisinger S, Heesch E, Krebs J, Holm R, Beck G, et al. Evaluation of pharmacokinetic properties and anaesthetic effects of propofol in a new perfluorohexyloctane (F6H8) emulsion in rats. A comparative study. Int J Pharm [Internet]. 2015;486(1-2):69-76. Available from: http://dx.doi.org/10.1016/j. ijpharm.2015.03.037

11. Thibaut J, Rivera T, Ahumada F. Anestesia endovenosa en perros mediante el uso de propofol en dosis única, premedicado con acepromazina-atropina y xilazina-atropina. Arch Med Vet [Internet]. 2002;34(1):25-35 [cited 2016 Nov 24]. Available from: http://www.scielo.cl/scielo.php?script=sci_arttext\&pid=S0301-732X2002000100003\&lng=en\&nrm=iso\&tlng=en

12. Zorrilla-Vaca A, Escandón-Vargas K, Brand-Giraldo V, León T, Herrera M, Payán A. Bacterial contamination of propofol vials used in operating rooms of a third-level hospital. Am J Infect Control [Internet]. 2016;44(1):e 1-3. Available from: http:// dx.doi.org/10.1016/j.ajic.2015.08.009

13. Cho J, Cho JC, Lee $P$, Lee $M$, Oh E. Formulation and evaluation of an alternative triglyceride-free propofol microemulsion. Arch Pharm Res. 2010;33(9):1375-87.

14. Dubey PK, Kumar A. Pain on injection of lipid-free propofol and propofol emulsion containing medium-chain triglyceride: A comparative study. Anesth Analg. 2005;101(4):1060-2.

15. Nakane $M$, Iwama $H$. A potential mechanism of propofol-induced pain on injection based on studies using nafamostat mesilate. $\mathrm{Br} J$ Anaesth. 1999;83(3):397-404.

16. Allchurch LG V, Crilly H. Fixed drug eruption to propofol. Anaesth Intensive Care. 2014;42(6):777-81.

17. Graham LF, Torres SMF, Jessen CR, Horne KL, Hendrix PK. Effects of propofol-induced sedation on intradermal test reactions in dogs with atopic dermatitis. Vet Dermatol. 2003;14(3):167-76.

18. McHale SP, Konieczko K. Anaphylactoid reaction to propofol. Anaesthesia. 1992;47(10):864-5.

19. Li X, Zhang $Y$, Fan $Y$, Zhou $Y$, Wang $X$, Fan $C$, et al. Preparation and evaluation of novel mixed micelles as nanocarriers for intravenous delivery of propofol. Nanoscale Res Lett [Internet]. 2011;6(1):275. Available from: http://www. nanoscalereslett.com/content/6/1/275

20. Wallentine CB, Shimode N, Egan TD, Pace NL. Propofol in a modified cyclodextrin formulation: First human study of dose-response with emphasis on injection pain. Anesth Analg. 2011;113(4):738-41. 
21. Lang BC, Yang J, Wang Y, Luo Y, Kang Y, Liu J, et al. An improved design of water-soluble propofol prodrugs characterized by rapid onset of action. Anesth Analg. 2014;1 18(4):745-54.

22. Minghella E, Benmansour $\mathrm{P}$, Iff I, Senior JM, Mosing M. Pain after injection of a new formulation of propofol in six dogs. Vet Rec [Internet]. 2010;167(22):8667. Available from: http://veterinaryrecord.bmj.com/cgi/doi/10.1136/vr.c5736

23. SAGARPA. Especificaciones técnicas para la producción, cuidado y uso de los animales de laboratorio. [Internet]. Diario oficial de la federación. México; 1999. p. 57. Available from: http://www.senasica.gob.mx/?doc $=743$

24. Bayne KAL. Environmental Enrichment of Nonhuman Primates, Dogs and Rabbits Used in Toxicology Studies. Toxicol Pathol. 2004;31 (1 Suppl):132-7.

25. Sumano H, Ocampo L. Farmacología veterinaria. 4th ed. México: Aranda; 2016.

26. World Health Organization. Stability testing of active pharmaceutical ingredients and finished pharmaceutical products, Annex 10. WHO Expert Comm Specif Pharm Prep. 2018;(52):309-51.

27. Martinez-Taboada F, Leece EA. Comparison of propofol with ketofol, a propofol-ketamine admixture, for induction of anaesthesia in healthy dogs. Vet Anaesth Analg [Internet]. 2014;41(6):575-82. Available form: http://dx.doi. org/10.1111/vaa. 12171

28. Bigby SE, Beths T, Bauquier S, Carter JE. Postinduction apnoea in dogs premedicated with acepromazine or dexmedetomidine and anaesthetized with alfaxalone or propofol. Vet Anaesth Analg. 2017;44(5):1007-15.

29. Bjur KA, Cannon BC, Fine AL, Ritter MJ, Schueler KE, Nemergut ME. Propylene Glycol Toxicity in Adolescent with Refractory Myoclonic Status Epilepticus. Case Rep Pediatr [Internet]. 2017;2017:1-3. Available from: https://www.hindawi. com/journals/cripe/2017/2979486/

30. Nanopolytox. Toxicological impact of nanomaterials derived from processing, weathering and recycling of polymer nanocomposites used in various industrial applications [Internet]. España; 2016. Available from: https://cordis.europa.eu/ project/rcn/94382/brief/es 